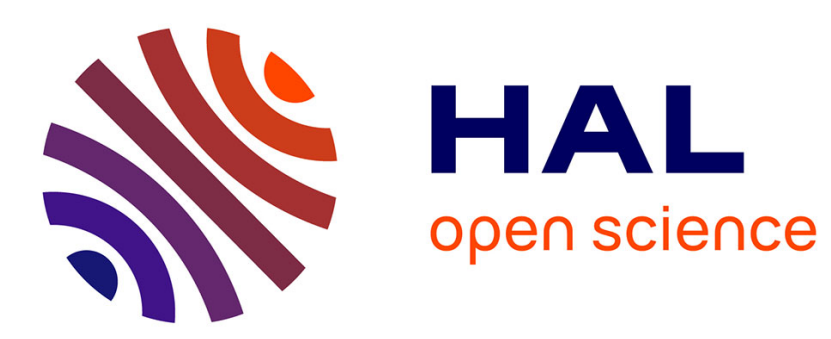

\title{
Malaisie. Le congrès mondial sur la langue malaise (Kuala Lumpur, 21-25 août 1995) \\ Daniel Perret
}

\section{To cite this version:}

Daniel Perret. Malaisie. Le congrès mondial sur la langue malaise (Kuala Lumpur, 21-25 août 1995). Bulletin de l'Ecole française d'Extrême-Orient, 1996, 83 (1), pp.349 - 356. 10.3406/befeo.1996.3800 . halshs-01880416

\section{HAL Id: halshs-01880416 https://shs.hal.science/halshs-01880416}

Submitted on 24 Sep 2018

HAL is a multi-disciplinary open access archive for the deposit and dissemination of scientific research documents, whether they are published or not. The documents may come from teaching and research institutions in France or abroad, or from public or private research centers.
L'archive ouverte pluridisciplinaire HAL, est destinée au dépôt et à la diffusion de documents scientifiques de niveau recherche, publiés ou non, émanant des établissements d'enseignement et de recherche français ou étrangers, des laboratoires publics ou privés. 


\section{Malaisie. Le congrès mondial sur la langue malaise (Kuala Lumpur,} 21-25 août 1995)

\section{Daniel Perret}

\section{Citer ce document / Cite this document :}

Perret Daniel. Malaisie. Le congrès mondial sur la langue malaise (Kuala Lumpur, 21-25 août 1995). In: Bulletin de l'Ecole française d'Extrême-Orient. Tome 83, 1996. pp. 349-356;

doi : https://doi.org/10.3406/befeo.1996.3800

https://www.persee.fr/doc/befeo_0336-1519_1996_num_83_1_3800

Fichier pdf généré le 07/05/2018 


\section{MALAISIE}

\section{Le Congrès mondial sur la langue malaise (Kuala Lumpur, 21-25 août 1995)}

Le congrès s'est tenu du 21 au 25 août 1995 dans les salons de l'hôtel Hilton de Kuala Lumpur. Organisé de main de maître par le Dewan Bahasa dan Pustaka sous la houlette de son dynamique nouveau directeur, A. Aziz Deraman, il a réuni près de 700 participants et a permis d'écouter 135 communications. Inauguré par le vice-Premier Ministre de Malaisie Dato' Seri Anwar Ibrahim, cet événement a bénéficié d'une importante couverture médiatique. Des représentants de 25 pays ont donné des communications, la Malaisie comptant à elle seule 69 communications, suivie par l'Indonésie avec 12 communications. L'Europe était représentée par 21 intervenants dont une délégation russe forte de 6 membres. Au cours de la matinée d'ouverture, des accords de coopération ont été signés entre le Dewan Bahasa dan Pustaka et 11 institutions étrangères dont l'École française d'Extrême-Orient.

Qu'un congrès d'une telle envergure demandant de très importants soutiens financiers ait eu lieu à Kuala Lumpur révèle le double enjeu que représente la langue malaise pour les Malaysiens. Le premier, international, était le thème officiel du congrès: bahasa melayu sebagai bahasa antarabangsa : wawasan dan keyakinan (la langue malaise comme langue internationale : vision et conviction).

D'entrée un constat général a été fait : malgré ses 250 millions de locuteurs le bahasa melayu n'a pas encore atteint le statut de langue internationale. À de nombreuses reprises il a été fait référence à l'âge d'or des sultanats lorsque le bahasa melayu était une véritable lingua franca. Ainsi dans son allocutation d'ouverture le vice-Premier Ministre Dato' Seri Anwar Ibrahim a rappelé :

«Nous devons utiliser la langue malaise comme l'ont utilisée les grands penseurs de notre peuple, de Hamzah Fansuri et Nuruddin al-Raniri jusqu'à Abdullah Munshi et Za'ba (......). Les grands penseurs de l'époque des sultanats malais ont bâti et forgé leur gloire dans leurs domaines respectifs en faisant d'une langue pauvre véhicule de mythes, une grande langue qui élargit l'horizon de pensée de la communauté et fait entrer dans l'arène de la pensée universelle».

La place essentielle prise par le bahasa melayu à l'époque des sultanats reposait sur un contexte économique florissant et la position de ces États comme carrefours commerciaux internationaux.

«À une certaine époque la langue malaise a été la lingua franca de cette région justement en raison de la position géopolitique des sultanats malais et de sa fonction dans le commerce régional et international » (Anwar Ibrahim).

Or, après la période coloniale qui a mis sous l'éteignoir ce rôle international du bahasa melayu, le temps revient d'un contexte économique et politique florissant pour le monde malayophone, à l'image de celui qu'avaient connu les sultanats. Ce renouveau économique et politique est perçu comme le signe annonciateur d'une renaissance du bahasa melayu comme lingua franca.

«Nous avons réussi à faire de la Malaisie, un pays autrefois écarté de la politique internationale, une nation aujourd'hui plus respectée. Nous avons accéléré le succès économique et faisons aujourd'hui figure de modèle de développement pour les pays en voie de développement. (......) Il semble qu'au milieu des passions tumultueuses soulevées 
aujourd'hui par la langue malaise, nous devons réfléchir un instant et formuler les mesures qui doivent permettre à la pensée, à la culture et à la science de progresser à l'unisson avec l'économie et la politique » (Anwar Ibrahim).

«Le secteur privé (malais) doit se représenter comme l'incarnation des marchands malais d'autrefois. (...) Leur rôle est très important et s'il est associé à une politique ferme du gouvernement, le prestige de la langue malaise comme lingua franca reviendra » (Hanapi Dollah, Universiti Kebangsaan Malaysia).

Parmi les propositions faites en séance pleinière le dernier jour du congrès sur le rôle international que peut et doit jouer le bahasa melayu dans l'avenir trois niveaux d'internationalisation ont été projetés : le bahasa melayu comme langue officielle de communication entre les nations du monde malayophone, le bahasa melayu comme langue officielle de l'ASEAN puisque $60 \%$ de la population de l'ASEAN est malayophone, le bahasa melayu comme langue officielle aux Nations unies puisque le nombre de locuteurs est supérieur au nombre de locuteurs parlant certaines langues officielles de cette institution internationale. C'est le cas du français.

Cette interrogation sur la place du bahasa melayu au niveau international a été en réalité reléguée au second plan par un autre enjeu, national celui-là. En fait le congrès a été surtout une occasion supplémentaire et exceptionnelle offerte aux Malaysiens de s'interroger sur la situation et le développement du bahasa melayu dans le contexte national malaysien et ceci peu de temps après une déclaration du Premier Ministre sur la distinction qu'il convenait maintenant d'observer entre le bahasa melayu et le bahasa malaysia, déclaration qui avait provoqué un certain émoi dans les milieux intellectuels malais.

Parmi les «isu » évoquées celle concernant l'usage du bahasa melayu dans le secteur privé et notamment dans les instituts d'enseignement privé de haut niveau est revenue à plusieurs reprises sur le devant de la scène :

«Nous ne pouvons pas dévier du principe que le malais soit la langue dans les affaires officielles et dans les relations entre le secteur privé local et le gouvernement. Nous ne nous laissons pas aller à abandonner ce principe devant l'insistance d'une élite coupée de ses racines culturelles. » (Anwar Ibrahim).

«Si de nombreux instituts privés d'enseignement de haut niveau qui existent aujourd'hui dans le pays privilégient dans l'avenir l'anglais ou le mandarin du fait que la plupart d'entre eux ont leur maison-mère en Grande-Bretagne, en Chine ou à Taiwan, alors le rôle du malais va diminuer de plus en plus dans le domaine scientifique » (Tan Sri Ismail Hussein, Berita Harian 23/08/95).

«Il est clair que dans les instituts privés d'enseignement de haut niveau, le malais n'occupe pas la place principale» (Dr. Nik Safiah Karim, Présidente Persatuan Linguistik Malaysia).

Cette «isu» a trouvé son point d'orgue lors du discours de clôture du congrès par le ministre de l'Éducation malaysien, Datuk Seri Mohd Najib Tun Razak. Il saisit l'occasion pour démentir la rumeur concernant la diffusion d'une circulaire ministérielle autorisant l'usage de l'anglais dans les pusat pengajian tinggi (IPT) pour les enseignements scientifiques et techniques.

La majorité des participants malaysiens ont été d'accord pour affirmer que le problème ne réside pas dans une soi-disant menace de l'anglais mais plutôt dans la trop fréquente méconnaissance de leur propre langue nationale par les Malaysiens.

«La vision réalisable concernant le malais est d'en faire définitivement la langue nationale et une langue scientifique, pas seulement le marqueur identitaire de la société malaise. (......) Ce projet ne peut réussir que si tous ou tout au moins une majorité de Malaisiens sont prêts à accepter le malais comme seule langue nationale. »( Abdullah Jumain Abu Samah, UKM).

Le Japon a été cité en exemple comme pays ayant réussi à utiliser sa langue comme un outil de progrès et par là à préserver son identité (Datuk Hassan Ahmad). À nouveau référence 
a été faite à l'âge d'or. Ainsi dans son discours d'ouverture Anwar Ibrahim a cité un long passage du Hikayat Hang Tuah concernant l'apprentissage de 12 langues étrangères par ce dernier. Un autre exemple a été fourni lors de l'intervention de Hanapi Dollah (UKM) affirmant qu'il n'y a pas lieu de s'inquiéter du désir du gouvernement malaysien à développer l'usage de l'anglais dans les Institusi Pengajian Tinggi. En effet une telle situation ne ferait que répéter celle qu'a connue Srivijaya où le sanscrit était utilisé dans les hautes institutions d'enseignement religieux. Par ailleurs, à l'époque de Melaka et d'Aceh, l'arabe était utilisé pour l'enseignement religieux à haut niveau.

Le caractère éminemment politique du congrès a quelque peu éclipsé son aspect scientifique d'autant que le temps dévolu à chaque communication était bien souvent trop court pour pouvoir esquisser un véritable débat collectif. La pléthore de communications a obligé les organisateurs à aménager quatre salles fonctionnant simultanément. Pour faciliter le choix des auditeurs le Dewan Bahasa avait réussi le tour de force de fournir à chacun des intervenants la grande majorité des communications en deux volumes reliés et ceci dès l'ouverture du congrès. Il n'est bien sûr pas question ici de commenter chacune des communications. Afin de donner une idée générale sur le contenu du congrès, nous récapitulons ci-dessous les thèmes abordés selon l'origine géographique des intervenants.

\begin{tabular}{|c|c|c|c|c|c|}
\hline \multirow[t]{2}{*}{ THÈME } & \multicolumn{5}{|c|}{ ORIGINE DES INTERVENANTS } \\
\hline & Malaisie & Indonésie & Europe & SEA & Autres \\
\hline Linguistique / Lexicologie & 14 & 3 & 2 & 2 & 3 \\
\hline $\begin{array}{l}\mathrm{BM} \text { (Bahasa Melayu): situations } \\
\text { régionales ou locales }\end{array}$ & & 4 & 5 & 8 & 2 \\
\hline Sociolinguistique & 14 & & 1 & & \\
\hline $\begin{array}{l}\text { Bahasa Melayu } \\
\text { Language scient. et tech. }\end{array}$ & 4 & & & 1 & \\
\hline BM et tech. de l'information & 2 & & 1 & 1 & \\
\hline Enseignement du BM & 3 & & 1 & 3 & 2 \\
\hline Internationalisation du BM & 12 & 3 & 1 & & 3 \\
\hline BM et histoire Nationale & 2 & & & & 1 \\
\hline Jawi & 1 & & & & \\
\hline Traduction & 2 & & 1. & 1 & \\
\hline Littérature traditionnelle & 2 & & 4 & 1 & \\
\hline Littérature moderne & 13 & 2 & 5 & 3 & 1 \\
\hline Langue vietnamienne & & & & 1 & \\
\hline & ---- & ---- & ---- & ---- & ---- \\
\hline
\end{tabular}

Pour terminer nous soulignerons que le Dewan Bahasa avait aussi très bien fait les choses du côté de la bonne chère et des divertissements puisque, chaque jour, les participants ont été conviés à déjeuner dans les salons du Hilton et les soirées ont été marquées par des réceptions à l'échelle du congrès. Chacune de ces receptions a bénéficié du soutien d'un ministère ou d'une grande société locale (ministère de l'Éducation, ministère de la Culture, journal Utusan Malaysia, Administration du Pèlerinage (Tabung Haji), Sistem Televisyen Malaysia Berhad et Datuk Bandar de Kuala Lumpur).

Daniel PERRET 
LISTE DES COMMUNICATIONS (par ordre alphabétique des intervenants)

\author{
A. Aziz Deraman (ML) \\ Pembinaan dan Pengembangan Bahasa \\ Melayu: Wawasan dan Keyakinan
}

Abdul Hamid Mahmood, Prof. Dr. (ML)

Cabaran dan Harapan Bahasa Melayu Sebagai

Bahasa Ilmu

Abdullah Hassan, Prof. Dr (ML)

Isu-Isu Mengenai Fungsi Bahasa Melayu

Abdullah Jumain Abu Samah (ML)

Bahasa Melayu Ke Arah Bahasa Antarabangsa : Sejarah, Penggayaan Leksikon dan Wawasan

Abdullah Tahir, Prof. Madya (ML)

Novel-Novel Mohd. Affandi Hassan: Meneliti

Kemungkinan Penerapan Konsep «Persuratan

Baru Melayu»

Abdulroya Panaemalae (TH)

Kedudukan dan Kewibawaan Bahasa Melayu di

Wilayah Sempadan Selatan Thailand:

Renungan Sejarah Silam dan Ramalan Arah

Tuju Masa Depan

Adelaar, Dr. Alexander K. (AU)

Malay In The Cocos (Keeling) Islands

Ahmad Kamal Abdullah (ML)

Puisi Melayu Mutakhir Di Tengah-Tengah

Perkembangan Puisi Dunia: Sebuah Lanskap

Bisu

Ahmad Samin Siregar, Drs. (IN)

Mozasa, Sasterawan Angkatan Pujangga Baru:

Pejuang Bahasa Dan Sastera Indonesia Yang

Gigih

Ahmad Zaki Abu Bakar, Dr. (ML)

Pengurusan Organisasi Cemerlang Berasaskan

Bahasa

Ajid Che Kob, Prof. Madya (ML)

Pembentukan Istilah Bahasa Melayu dan

Pengglobalisasian Penggunaannya

Alieva, Natalia F. (RU)

Perkembangan Struktur Bahasa Melayu:

Imbangan Sarana Analitis dan Sintetis Pada

Berbagai Tahap Sejarah

Amat Juhari Moain, Prof. Dr. (ML)

Tulisan Jawi Sebagai Wahana Pelestarian

Bahasa, Budaya dan Tamadun Dunia Melayu
A. Rahman Hanafiah et Mana Sikana, Dr. (ML)

Sastera Melayu Pasca-Modenisme: Dari Dunia

Yang Terpendam ke Alam Pencerahannya

Asmah Haji Omar, Prof. Dato' Dr. (ML)

Kajian Bahasa Melayu Dalam Usaha

Mempertingkatkan Mutu Pengajaran Bahasa

Melayu

A. Wahab Ali, Prof. (ML)

Suara Sasterawan Dalam Arus Pembangunan

Awang Mataim Bakar (BR)

Bahasa Melayu Dalam Sistem Pendidikan

Dwibahasa Negara Brunei Darussalam

Awang Sariyan (ML)

Pengantarabangsaan Bahasa Melayu Melalui Institusi Pendidikan Tinggi: Catatan Tentang Pengalaman Universiti Islam Antarabangsa Malaysia

Ayob Yamin dan Zakaria Ariffin (ML)

Bahasa Melayu Sebagai Wahana Pengucapan Sastera: Pengalaman Penulis-Penulis Bukan Melayu Di Malaysia

Azhar M. Simin dan Abd. Aziz Idris, Prof. Madya Dr. (ML)

Dari Bahasa Pasang ke Bahasa Tukang:

Memanfaatkan Pandangan Pemimpin

Badaruddin Pengiran Hj. Ghani, Pengiran Dato' Paduka (BR)

Peranan Dewan Bahasa dan Pustaka Dalam Pembinaan Dan Pengembangan Bahasa Dan Sastera Melayu Brunei

Bahren Umar Siregar, Dr. (IN)

Penafsiran Semantik Terhadap Makna Kalimat: Mencari Tipologi Semantik Bahasa Nusantara

Bambang Suhartono Mohd. Said, Drs. (ML) Bahasa Melayu dan Cabaran Global: Antara Kewibawaan vs. Sikap

Braginsky, Prof. Dr. Vladimir I. (UK)

Tarikh Penciptaan Sejarah Melayu: Sebuah Pertimbangan Semula

Chaiyanara Paitoon, Dr. M. (SG)

Kata-kata Sanskrit dalam Bahasa Melayu dan Bahasa Thai: Satu Kajian Perbandingan dari Segi Fonologi

Chambert-Loir, Dr. Henri (FR)

Wawasan Politik Sedunia Dalam Hikayat Hang Tuah 
Choy Kim Chuah (ML)

Bahasa Melayu Yang Bertaraf Antarabangsa

Chung Young-Rhim, Prof. Dr. (KO)

Perbandingan Sastera: Imej Manusia MelayuKorea Dalam Novel Awal

Collins, Prof. James T. (US)

Bahasa Melayu Sebagai Bahasa Antarabangsa :

Antara Kenyataan dan Keraguan

Darwis Harahap, Dr. (ML)

Mencari Sifat Bahasa Melayu Yang Sederhana

Dorofeeva, Tatiana V. (RU)

Pengajaran dan Pembelajaran Bahasa Melayu di Rusia

Duc Ninh Nguyen, Prof. Dr. (VN)

Bahasa Vietnam-Bahasa Kebangsaan dan

Cabarannya

Fang Wei Bang $(\mathrm{CH})$

Pengalaman Siaran Bahasa Melayu Radio

Antarabangsa China

Faridah Hj Abdul Hamid, Dayang (BR)

Perkembangan Penulisan Cerpen di Brunei

Selepas Kemerdekaan

Farid M. Onn, Prof. Dr. (ML)

Mengantarabangsakan Bahasa Melayu: Nota

Kepada MABBIM

Fatimah Hj. Awang Chuchu (BR)

Cabaran Menjelang Abad Ke-21: Peranan

Bahasa Melayu di Negara Brunei Darussalam

Fitriaty Harahap, Drs. (IN)

Perkembangan Bahasa Melayu di Tanah Batak

Ghoshal Baladas, Prof. (ID)

Programmes For The Development of

Malay/Indonesian Language: The Experience of India

Grijns, Dr. C.D. (NT)

A Corpus of Malay: A Challenge to Linguists, Information Scientist and Administrators

Halimah Haji Ahmad (ML)

Penggunaan Bahasa Melayu Di Kalangan Penutur Asing Bahasa Melayu: Satu Kajian Sikap

Hamza Mustafa Njozi (TZ)

Projection in Folklore: The Case of Hikayat

Amir Hamzah
Hanapi Dollah, Dr. (ML)

Bahasa Melayu dan Globalisasi: Sejarah dan Keyakinan

Harimurti Kridalaksana, Prof. Dr. (IN)

Pengembangan Ilmu Bahasa Sebagai Upaya

Peningkatan Apresiasi Terhadap Bahasa

Melayu

Harun Salim (ML)

Imej Bahasa Melayu Dalam Industri Perumahan dan Hartanah

Hashim Awang, Prof. Dr. (ML)

Retorika Melayu dari Tanggapan Za'ba

Hashim bin Haji Abd. Hamid, Dr. (BR)

Almarhum Sultan Omar Ali Saifuddien,

Sasterawan Diraja Brunei

Hashim Musa, Prof. Madya Dr. (ML)

Pengintelektualan dan Pendaulatan Bahasa

Malaysia Sebagai Bahasa Pertama : Cabaran

Globalisasi Maklumat, Pendidikan dan

Teknologi

Hoffman, Dr. John E. (AU) dibentangkan oleh Prof. Clive Kessler

Sumpah Pemuda : International Malay On Oath

Huang Chengfang, Prof. $(\mathrm{CH})$

Sifat Kefuzian Makna Kata Melayu

Husen Abas, Prof. Dr. (IN)

The Malay Languages: From A Lingua Franca to Become a Language of International

Communication (Indonesian Experience)

Ibrahim Ahmad Bajunid, Dr. (ML)

Pembinaan dan Penggunaan Bahasa Melayu

Tinggi Dalam Usaha Pembinaan Tubuh Ilmu

Peribumi Bidang Pengurusan Pendidikan

Ilyas Mahyudin, Drs. (TH)

Upaya Memasyarakatkan Pemakaian Bahasa

Melayu Baku di Selatan Thailand

Ismail Dahaman (ML)

Beberapa Patokan Tentang Potensi Aspek

Ekonomi Sebagai Pemangkin Bagi

Pengantarabangsaan Bahasa Melayu

Ismail Salleh (ML)

Citra Bahasa Melayu Dalam Usia 39 Tahun

Perancangan Bahasa: Satu Penilaian

Jones, Dr. Russell (UK)

Malay: On the International Components of an International Language 
Kamal Shukri Abdullah Sani (ML)

Bahasa Melayu: Kewajaran Istilah Dan

Sebutan Namanya

Kamsiah Abdullah, Dr. (SG)

Perkembangan Pendidikan Bahasa Melayu di

Singapura Dengan Rujukan Khusus Tentang

Latihan Guru-Guru Melayu

Khaidzir Hassan (ML)

Aspek Kohesi dalam Novel A. Samad Said

Sepanjang Dasawarsa Enam Puluhan

Khathijah Abd. Hamid (ML)

The Use Of The Malay Language In Education And Society In Malaysia

Koh Young Hun, Prof. Madya Dr. (KO)

Dunia Melayu Yang Tersirat Dalam Tetralogi

Bumi Manusia - Pramoedya

Kukushkin Serguei V. (RU)

Koleksi Bahasa Melayu Di Perpustakaan

Negara Rusia (Pusat Dokumentasi Asia dan

Afrika)

Le Roux, Dr. Pierre (FR)

A Little-Known Treasure of Malay Culture: The Oral Literature of Patani (Patani, Yala and Narathiwat Provinces, Southern Thailand)

Littrup, Dr. Lisbeth (DM)

The Car In The Short Stories By Anis Sabirin

Lodel, Dr. Hans-Heinrich (GR)

Pengalaman Penterjemah Mengerjakan Karya-

Karya Sastera Melayu ke Bahasa Jerman

Mangantar Simanjuntak, Dr. (BR)

Pengutamaan Bahasa Melayu dalam Semua

Bidang, termasuk Sains dan Teknologi,

Menjamin Potensinya sebagai Bahasa Ilmu dan

Bahasa Supranasional di samping menjamin

Pembangunan Negara Yang Maksimum

Md. Shahri Pehin Orang Kaya Jurulateh Adat Istiadat Diraja Awang Haji Md. Hussin, Awang (BR)

Pengembangan Bahasa Dan Sastera Melayu:

Pengalaman Brunei Darussalam

Mason-Hoadley, Dr. C. (SW)

"The Malay Dilemma» In The Malay Novel:

Urban Readers And rural Settings

Mat Rofa Ismail, Dr. (ML)

Bahasa Melayu Ilmiah: Pengkaryaan Perubatan Tradisi, Falak dan Kosmologi Dalam Bahasa Melayu
Mohamed Anwar Omar Din (ML)

Pembaratan Dalam Bahasa Dan Kesusasteraan Melayu

Mohd. Kassim Ismail (ML)

Pendidikan Kesusasteraan Dalam Mata

Pelajaran Bahasa melayu Kurikulum Bersepadu

Mohd. Noh Nyata (ML)

Berbahasa Jelek - Cara Berbahasa Yang

Menjejaskan Keterampilan Berbahasa Melayu.

Patutkan Keadaan Ini Dibiarkan Berterusan?

Mohd Salleh Abd. Majid, Dato' (ML)

Penyebarluasan Bahasa Melayu Dalam Sektor

Kewangan

Mohd. Samsudin (ML)

Bahasa Melayu dan Cabaran Global: Menilai

Peranan Bahasa Melayu Dahulu, Kini dan Akan

Datang di Pentas Antarabangsa

Muhammad Haji Salleh, Prof. Dr. (ML)

Yang Indah-Indah Estetika Sastera Melayu

Muhammed Haron, Drs. (SA)

Foreign Language Teaching in South Africa:

Making a Case For Bahasa Melayu

Mus Chairil Samani dan Cik Nor'aini Sayati

(ML)

Keterbacaan Teks Akademik Berbahasa Melayu

Nagasura T. Madale, Prof. Dr. (PH)

Malay Studies Programme In The Philippines:

Problems, Issues And Prospects

Nasrun Alias (ML)

Perkembangan dan Kesinambungan Ilmu

Sintaksis di Malaysia: Tinjauan Terhadap

Aliran dan Tokoh

Nguyen Huy Can, Dr. (VN)

Bahasa Melayu with the Lingual-Cultural

Contact In Viet-Nam

Nik Safiah Karim, Prof. Dr. (ML)

Pengajian Tinggi dan Pengantara-bangsaan

Bahasa Melayu

Noresah Baharom (ML)

Perubahan dan Inovasi Leksikal Dalam Bahasa Melayu: Unsur Penerimaan dan Kelangsungan Hidup

Nor Hashimah Jalaluddin, Dr. (ML)

Ganti Nama Melayu: Satu Analisis Fungsi Dan Pola 
Norizan Abd. Razak (ML)

Pengajaran Bahasa Melayu Berbentukan Komputer: Cabaran dan Implikasinya di dalam pengembangan Bahasa

Norliza Hani Md. Ghazali et al. (ML)

Peranan Hubungan Semantik: Pindahan Makna Di Dalam Terjemahan

Normah Abdullah dan Mat Nor Hussin (ML)

Penginter-nasionalan Bahasa dan Budaya Melayu Dalam Bahan Pengajaran Bahasa

Untuk Pelancong

Norsimah Mat Awal (ML)

Talk Ni Sure Best Punya: Penggunaan Leksikal Bahasa Inggeris Di Dalam Pertuturan Bahasa Melayu

Nothofer, Prof. Dr. Bernd (GR)

Pengajian Dialek di Bangka dan Sumbangannya Pada Pembinaan Sejarah Penyebaran Penutur Bahasa Melayu Dan Bahasa Barito Tenggara

Nurdin Langgole, Dr. M.S. (IN)

Kekerabatan Bahasa Melayu dan Bahasa

Indonesia : Suatu Pendekatan Leksikostatistik

Nur Nina Zuhra, Prof. Dr. (ML)

Bosnia: A Turning Point of Contemporary

Malay Poetry

Ogloblin, Prof. A.K. (RU)

Bahasa dan Budaya Melayu di Saint Petersburg

Oyong Karmayuda (IN)

Peranan Bahasa Melayu Dalam Pengembangan Pencak Silat

Parnickel, Dr. Boris (RU)

The Development of Malay Literature In The Context of Other Literatures Of Nusantara (9th to 19th Centuries)

Perret, Dr. Daniel (FR)

Kota Raja Dalam Kesusasteraan melayu Tradisional: Citra dan Sumbangan Untuk Sejarah Kota Lama Di Dunia Melayu

Ramlah Adam, Prof. Madya Dr. (ML)

Pendaulatan Bahasa Melayu Sebagai Bahasa

Kebangsaan Negara: Peranan Golongan

Nasionalis Dari Tahun 1930an Sehingga 1957

Ramli Isin, Prof. Madya Dr. (ML)

Hashim Awang Dalam Konteks Perkembangan Esei dan Kritikan Sastera Melayu Moden
Ramli Md. Salleh, Dr. (ML)

"Ada sebagai Kata Kerja dalam Bahasa

Melayu

Razali Kassim, Drs. (IN)

Sastera Melayu Raya : Perspektif dan Realiti

Ridwan, Prof. Dr. HTA (IN) Disampaikan oleh

H. Ahmad Samin Siregar, Drs

Bahasa Melayu Sebagai Bahasa Pergaulan

Dunia: Tentangan Dan Kenyataan

Rogayah A. Razak (ML)

Kuantifikasi di dalam Bahasa Melayu daripada

Sudut Sintaksis dan Semantik - Satu Pendekatan

Kuasaan dan Tambatan

Roksana Bibi Abdullah (SG)

Analisis Kompenential Istilah-Istilah Dalam

Bidang Sintaksis

Ruzy Suliza Hashim (ML)

Pengolahan Semula Tun Fatimah: Dua

Perspektif

Sahlan Mohd. Saman, Prof. Madya Dr. (ML)

Gaya Bahasa Penyair Muda: Suatu Pengamatan Kesan

Sanat Md. Nasir (ML)

Yang Terabai dalam Penulisan Tatabahasa

Bahasa Melayu

S. Faizah Soenoto Rivai, Prof. Madya (IT)

Kata-kata Melayu Dalam Kamus Bahasa Italia (Sebuah Catatan)

Shaik Noor Alam S.M. Hussain, Dr. (ML) Bahasa Melayu Dalam Undang-Undang Membentuk Dimensi Global

Shaharir Mohamad Zain dan Wan Ramli Wan Daud, Prof. Dr. Madya (ML)

Bahasa Melayu Dalam Sains dan Teknologi:

Mitos, Kenyataan dan Unggulannya

Sheikh Othman Sheikh Salim (ML)

Keupayaan Kata Dan Kewibawaan Bahasa

Shoho Isamu, Prof. Madya Dr. (JP)

I-lowering in Malay Focus Fronting

Constructions

Sikorsky, Prof. Wilen.V. (RU)

Wordsworth Dari Singapura

Siti Khaotijah Mohammad et al. (ML)

Bahasa Melayu Dahulu lawan Bahasa Melayu

Sekarang dari segi Penggunaan dan Pedoman: 
Satu Kajian Kes Perbandingan di antara Penggunaan dalam Hikayat Hang Tuah dengan Pedoman dalam Tatabahasa Dewan

Siti Zainon Ismail, Prof. Madya Dr. (ML) Bahasa Dalam Kehidupan Seni Budaya: Satu Kajian Istilah Seni dan Teknologi Wilayah Alam Melayu

Sukapiring, Drs. P. (IN)

Dialek Bahasa Melayu/Indonesia di Kotamadya Medan

Supki Haji Sidek dan Puan Aisha Hussain (SG)

Pendekatan Genre Dalam Pengajaran

Kemahiran Menulis di peringkat Sekolah

Menengah

Syed Omar Syed Mohamad (ML)

A Technical Form Of Malay Language (Malay Numerical Classifiers)

Syed Othman Syed Omar, Pr. Madya Dr. (ML)

Ledakan Kemahuan Dalam novel Melayu Mutakhir

Teh Gallop, Annabel (UK)

Malay Comic Books From The 1950's and 1960's

Teoh Boon Seong, Dr. (SG)

Teknologi Maklumat dan Perkembangan Bahasa Melayu

Teo Kok Seong, Dr. (ML)

Bahasa Melayu Sebagai Bahasa Antarabangsa : Beberapa Pertimbangan Dari Sudut Ilmu Perancangan Bahasa

Toda Dami, N. (GR)

Kembali Ke Akar Identiti Nusantara Terhempas Kepada Abracadabra Poetry Reading

Tol, Dr. Roger (NT)

New Methods For The Dissemination of Malay Manuscripts

Ungku Maimunah Mohd. Tahir, Prof. Madya

Dr. (ML)

R.J. Wilkinson dan Pensejarahan Kesusasteraan Melayu Moden

Utari Sudewo, Drs. (IN)

Bahasa Melayu Sebagai Bahasa Pengantar:

Khususnya Penggunaan Kata Sapaan di

Jakarta, Ibu Kota Republik Indonesia

Vanicek, Dr. Eva (NZ)

Teaching Language Through Literature
Vasiljev, Prof. Dr. Ivo (CZ)

Going International (A Sociolinguistic Approach)

Wan Fuad Wan Hassan, Dr. (ML)

Peristilahan, Penterjemahan dan Penulisan Dalam Bahasa Melayu: Pengalaman Bidang Geosains

Wan Hashim Wan Teh, Prof. Dato' Dr. (ML)

Penggunaan Bahasa Melayu Di Institusi

Pengajian Tinggi: Kemajuan Dan Harapan

Wu Zong Yu, Prof. (CH)

Kumpulan Istilah-Istilah Melayu Nusantara

Pada Abad Ke 15 - Satu Kajian Awal

Yayah B. Lumintaintang, Dr. (IN)

Program Pembinaan dan Pengembangan

Bahasa di Indonesia

Yang Quee Yee (SG)

Penterjemahan Bahan-Bahan Bahasa Melayu Di Bidang Bahasa Tionghua - Satu Pengkajian Antara Tahun 40'an - 60'an

Zaharani Ahmad (ML)

Analisis Fonologi Deretan Vokal Bahasa Melayu Daripada Derivasi Rumus Kepada Tatatingkat Kekangan

Zaharin Yusoff, Prof. Dr. (ML)

Teknologi Maklumat dan Bahasa Melayu: Ke Arah Sebuah Pusat Maklumat Bahasa Melayu

Zahrah Abdul Ghaffur, Dr. (ML)

Artikulasi: Topik dan Fokus Dalam Bahasa Melayu

Zeus Salazar, Prof. (PH)

Perspectives On Malay/Indonesian in The Philippines

Zhou Nanjing, Prof. (CH)

Bahasa Melayu/Indonesia Bisa Jadi Bahasa Internasional

Zulkifley Hamid (ML)

Pengajaran Bahasa Melayu: Apakah Yang Seharusnya Diajar? 